\title{
Statement and Self-Management Analysis in Mountain Minorities Southeast Chinese Elderly with Chronic Pain
}

\author{
Juan Huang ${ }^{1}$, Ting Sun ${ }^{2}$, Ziping Huang ${ }^{3}$, Zuoqin Liu ${ }^{3}$ \\ ${ }^{1}$ Department of Foundation Nursing, Youjiang Medical School for Nationalities, Baise, China \\ ${ }^{2}$ Department of Emergency, Affiliated Hospital of Youjiang Medical School for Nationalities, Baise, China \\ ${ }^{3}$ Department of Clinical College, Youjiang Medical School for Nationalities, Baise, China
}

Email address:

214336856@qq.com (Juan Huang)

\section{To cite this article:}

Juan Huang, Ting Sun, Ziping Huang, Zuoqin Liu. Statement and Self-Management Analysis in Mountain Minorities Southeast Chinese Elderly with Chronic Pain. Clinical Medicine Research. Vol. 7, No. 3, 2018, pp. 51-56. doi: 10.11648/j.cmr.20180703.11

Received: May 8, 2018; Accepted: May 29, 2018; Published: June 13, 2018

\begin{abstract}
Chronic pain (CP) is a very common problem in elders, due to bodily degenerations, worldwide. Studies carried out in various countries have shown that $\mathrm{CP}$ is associated with the elders' quality of life, significantly limiting their activities and hampering them to maintain an independence lifestyle. What's worse, elderly suffering from CP mostly also experience mental problems. Yet, there have been only a few such research done and reported on this topic, concerning the elderly ethnic montagnards in the rural southwest Guangxi of China. This study aimed to explore the statement and self-management of rural dwelling elders with CP. First, cross-sectional surveys were conducted and then interviews were carried out. 150 elder people experiencing $\mathrm{CP}$-- pain suffered at least 4 to 5 days a week during the past 3 months, according to the criteria of the international Association for the study of Pain (IASP) -- were enrolled in this study by convenience sampling. They were asked to fill in 3 questionnaires; the first related to participants' mental status, the second related to participants' perception of pain intensity, and the third related to pain's impact on participants' daily life. Following the completion of questionnaires, individual interviews were conducted, with the help of some students who are fluent in local native languages as well as in Chinese. The results show that CP significantly affected participants' quality of life. The prevalence of suffering from multifocal CP was $90 \%$. In the management of CP, $64 \%$ people mainly relied on paregoric means; a wide range self-management techniques were mentioned such as hot compress application for which some plant material was used, collected from the surrounding environment; only a few people went to seek professional treatment. Moreover, most of them said that they had reconciled to the pain or consider it as part of their fate. Encourage older people to seek different ways to manage their pain, not just traditional but complementary and professional approaches. In such severely lacking professional high-level medical resource environment, the elder people should change their cognition of $\mathrm{CP}$ and choose pertinence approaches and instruments based on their own condition.
\end{abstract}

Keywords: Chronic Pain, Self-Management, Mountain Nationalities of Southwest Guangxi of China, Chinese Elderly

\section{Introduction}

Chronic pain is a term used to describe pain that has persisted for 3 months or more, in accordance with the International Association for the Study of Pain definition.[1] According to the formal report of the Chinese government, it is estimated that $15.4 \%$ of older adults aged 60 years and over in the future 5 years, especially which live in the mountain rural area, the velocity is faster than city and town.[2] In the mountain rural area, elder people always live alone with the young grandchildren, the majority young people are outside for seeking money to support the family. Moreover, the economic level there is low, inconvenient transportation, short of physicians and nurse to provide professional help. Many older people accept pain as part of their life and do not seek help until pain become severe and unbearable. Zhou Wei [3]. investigated the Midwest rural area old people's quality of life showed that the main problems aging related are pain and disability.

As a result of chronic pain, older persons tend to mobilize in a slow and purposeful way with caution, mobility and levels of physical activity are significantly impaired, $[4,5]$ chronic pain and mobility difficulties interact in the older person, causing a 
negative feedback loop, in which reducing physical activity to avoid pain usually ends up further increasing pain when activity is undertaken. Thus chronic pain robs older people of their mobility level, and quality of life is adversely affected.

Management of chronic pain is variety in general. Since the first IASP (International Association For the Study of Pain Congress) congress, the field of pain medicine has seen a huge global evolution. 40 years after this first IASP conference, the pain medicine had developed much, which can help the pain patients relieve their pain [6] (conference of Italy). However, physicians are often reluctant to prescribe adequate analgesic treatment for fear of inducing drug addiction. It was found that $90 \%$ of analgesic medications were ordered on an as "needed" basis. Most patients, especially old people generally accept pain as part of growing old, and often fail to ask for pain relief despite experiencing severe pain and they rarely to request the analgesic over-the-counter. The majority of individuals using analgesic medications for chronic back pain reported less than adequate pain relief. Adverse effects from pain medications are common (66\%) for chronic back (CPB) pain sufferers. CBP sufferers, who report chronic pain for longer than 2 years, also detail using approximately 25\% more prescription analgesics than those who have experienced the pain for less than 2 years. The increase in the number of adverse side effects negatively impacts quality of life [7]. In this regard, the pain patients always use non-prescription, preferably non-pharmacological approaches, like chiropractic treatment including instrument-assisted for non-specific neck pain in community-dwelling older people [24], photomodulation in the treatment of chronic pain [25]. People with chronic pain frequently utilize both conventional and complementary and alternative medicine (CAM) [8], mountain specific therapies. Acupuncture and chiropractic care are considered the most highly accepted by physician groups massage therapy (MT), as one of the earliest and most primitive tools for pain, has been employed by most Chinese patients with neck pain, it might be at least immediately effective and safe, preliminarily recommended as a complementary and alternative treatment for patients with neck pain.

There is growing support for a shift in chronic pain management toward a patient-centered approach that accounts for the lived experience of the person, [9] In many chronic pain syndromes, the latter may exist even without a considerable nociceptive input; or even when there is some nociceptive input, this may not necessarily correlate with the patient's intense emotional experience of pain; hence the justification for the influence of psychological factors [10]. There is enough evidence from numerous clinical studies that the 'anxiety' status of a patient may be strongly associated with the intensity of the surgical pain experience, [11-15] it is recommended to use distraction to relieve pain as suggested by the gate control theory. Similarly, some study advocated that interprofessional treatment is recommended, and consequently interprofessional education in pain management is necessary [26], not only to the physic, but rather to the psychology. Cognitive behavioral strategies for pain management include hypnosis, relaxation with guided image, distraction, and the use of support groups. Distraction is one of the important uses of cognitive behavior techniques to relieve pain.

For the people who live in the rural mountain district, the economic level is low, inconvenience transportation, low education, short of medical resources. Individuals living with chronic pain face daily challenges of managing symptoms, modifying roles and responsibilities, and coping with the negative emotional consequences of pain. Self-management interventions teach a variety of strategies to meet these challenges and build participants' self-efficacy for their use.

The goal of the present study was to examine the statement of the old people live in rural mountain districts in southwest Guangxi of China with chronic pain and their self-management, in order to deliver more effective methods to help them through answering questions, providing advice, addressing barriers and facilitators, and encouraging self-management efforts.

\section{Subjects and Methods}

\subsection{Subjects}

The sample consisted of 150 older persons without dementia. All members met the criteria of (1) experienced chronic pain: persistent or intermittent pain at least 3 months, NRS score more than 1;(2) Age $\geq 60$ years old;(3) The score of Hasegava Dementia Scale $\geq 22$ (4) Excluded from participation in this study if they had vision problems, a history of psychiatric disorders, particular depression, alcoholism, cerebral trauma, transient ischemic attack, hydrocephalus, neoplasm, epilepsy, disturbances of consciousness or focal brain disorders.

\subsection{Methods}

\subsubsection{HDS-R (Hasegawa Dementia Rating Scale, HDS-R)}

$H D S-R$ (Hasegawa Dementia Rating Scale, HDS-R) the team used the HDS-R to measuring cognitive function levels to identify the participants. The Hasegawa dementia rating scale-revised (HDS-R) and mini-mental state examination (MMSE) Japanese edition are commonly used questionnaires to evaluate cognitive functions. The HDS-R was developed in 1991 and comprises nine questions; the possible score ranges from $0-30$, with lower scores indicating more severe dementia. It is well validated in Japan, with $93 \%$ sensitivity and $86 \%$ specificity [4].

\subsubsection{VAS (Visual Analogue Scales, VAS)}

VAS (Visual Analogue Scales, VAS) To assess pain intensity and pain affect, the visual analogue scales was administered. VAS is a line of $100 \mathrm{~mm}$ in length with zero representing no pain at one end and 100 representing pain as bad as it could be at the other end. A higher score indicates more pain. Participants were asked to report their pain intensity by indicating a point on the line. The VAS for the 
rating of pain has been validated for the Chinese population and has been found to be accurate and reliable [16-17]. To increase reliability in this research, pain assessment took place twice, with a time interval of approximately 1 month.

\subsubsection{BPI (Brief Pain Inventory, BPI)}

BPI (brief pain inventory, BPI) BPI (short form), BPI short form has been used since it is the standard for use within clinical and research settings [18] This version of the BPI measures the intensity of pain over a 24-hour time period, that is, the worst, least and average pain and the interference in seven particular domains such as sleep, activity and mood. It is an 11 -item tool with a series of $0-10$ scales, where $0=$ least interference/intensity. All BPI domains are documented as a value between 0 and 10 to allow for easy comparison between pre-post values [19]. Researchers used this questionnaire to evaluate the influence on the old people's daily life, the impact of pain on mood, relationships with other people, walking, sleeping, work, joy of life and leisure activities. The score range from $0 \sim 10$, the score higher, the more impact.

\subsection{Training}

The people who took responsible to delivery the questionnaire were all from local and knew the localism. However, they were not professional members, so the research team required them to attend one day training led by the research team members, at the same time, one research team member accompany one worker together went to the old people's house to finish questionnaire. The research team member help and monitored the process.

\subsection{Data Collection}

The research team delivered 150 questionnaires and took back 150 items, the ratio is $100 \%$. The data was collected by research members who are all from local village and familiar the localism. They mainly took responsibility to help the old people to finish the questionnaires and explained the questions.

The data collected included: sociodemographic data (age, sex, academic level and nationalities), the characteristics of the pain impact the life, measured by BPI, such as sleep, activity and mood. The self-management and self-efficacy about chronic pain.

\subsection{Statistical Analysis}

Several statistical methods were used in data analysis. Constituent ratio was described the strategy of self-management of old people. Data was displayed as Mean \pm SD also used to evaluate whether significant difference existed self-efficacy among different age groups. A $p$ value of $<0.05$ was considered statistically significant.

\section{Results}

\subsection{HDS-R Scores}

HDS-R scores that are 10 or lower (from a possible maximum score of 33) are considered indicative of dementia (sensitivity 0.90, specificity 0.82), 10.5-21.5 indicates pr-dementia; 22.0-30.5 indicates sub-normal; normal cognitive function is indicated by HDS-R scores of 31 or higher. According to the survey, participants' score met the normal cognitive was $35.4 \%$. However, most people belong to sub-normal level, was $64.6 \%$, the team tried to find the relationship between the scores and the duration of chronic pain, but there was no positive relation. This result strongly demonstrated that the chronic pain can reduce the cognitive ability to some extent, regardless of the duration of chronic pain.

Table 1. HDS-R Scores.

\begin{tabular}{lll}
\hline score rang & Case (n) & Percentage (\%) \\
\hline $22.0-30.5$ & 97 & 64.6 \\
$31-33$ & 43 & 35.4 \\
\hline
\end{tabular}

\subsection{Sociodemographic Data}

Totally 150 elderly residents ( 88 male and 62 female) of rural southwest Guangxi of China mountain area who were experienced chronic pain took part in the program. Their ages ranged was 60 to 91 years, the average age was 69 years. The nationalities distribution: 87 the Zhuang nationality, 45 the Han nationality, 3 the Tujia nationality, 5 the Miao nationality, 9 the Yao nationality, 1 the $\mathrm{Bu}$ Yi nationality, over $70 \%$ were national minority. In the terms of economic level, over half of the participants had not received any formal education, they were not able to read and write Chinese, they need the research members to explained to finish the questionnaires. 19 cases were primary school not finished, 78 had received primary educational level, 33 had received secondary education, and 19 had received high school educational level, only 1 case had received higher vocational training. $90 \%$ of them do the land job, while others were worker or street seller.

\subsection{General Areas and Intensity of Pain}

Among the individuals suffering CP, 90\% experienced pain at multiple locations, the most common sites being the limbs and low back. During the past 24 hours, the average score of pain intensity was 65 on 100-mm VAS. Most of the old people chose the cheap medication to relieve pain. $61.6 \%$ could reach the efficiency, however, $34.2 \%$ could not reach the relief efficiency, may related to the drug anti, due to long-term use.

\subsection{Impact of Daily Life}

Old person reported pain significant impact their daily life such as activity, sleep, mood, walking, normal work, joy and relationship with others. Nearly half of participants (5.49 \pm 2.76) identified sleep as one of the top one (Table 2). some participants reported in the daily life, they needed help in grooming and dressing, the CP prevented them from doing previously routine things; could not walk a long time due to the pain; reduction the opportunities of outside, felt separated from others and social. 
Table 2. Statement of chronic affect of daily life.

\begin{tabular}{lll}
\hline Items & Score range & $\mathbf{x} \pm \mathbf{s}$ \\
\hline Daily life & $0 \sim 9$ & $4.51 \pm 2.24$ \\
Motion & $0 \sim 10$ & $3.24 \pm 2.31$ \\
Walking & $0 \sim 10$ & $3.73 \pm 2.91$ \\
Work & $0 \sim 9$ & $4.01 \pm 2.33$ \\
Interpersonal Relationship & $0 \sim 9$ & $2.29 \pm 1.95$ \\
Sleep & $0 \sim 10$ & $5.49 \pm 2.76$ \\
Hobbies & $0 \sim 8$ & $2.85 \pm 2.26$ \\
\hline
\end{tabular}

\subsection{Self-Management of Chronic Pain}

The data (Table3) collected showed the management of chronic pain focus on the medication bought from pharmacy store. $64 \%$ people chose to use medication bought from the pharmacy store, most of them use aspirin; $14.7 \%$ old people chose physical therapies, common strategies included application of Chinese herbal oil, self massage, cold or warm compression, using medical pads, these strategies they heard from relatives and friends or traditional Chinese practitioner, $12.67 \%$ old people chose other approaches, however, no one sought treatment or help from physicians just solely for the CP. They just consult physicians about their $\mathrm{CP}$ at the time when they had follow-up and consultation for other systemic problems. In the other approaches, the team did not find someone seek mental support from their family, many old people accepted the CP as part of their life, and did not take medication until severe intolerance pain happened.

Table 3. Statement of self-Management.

\begin{tabular}{lll}
\hline Strategies & Cases (n) & Percentage (\%) \\
\hline Medication & 96 & 64.0 \\
Physiotherapy & 22 & 14.7 \\
Herbal compression & 13 & 8.67 \\
Others & 19 & 12.67 \\
\hline
\end{tabular}

\section{Discussion}

The present study demonstrated a decreased in pain perception and influenced significantly quality of life among the old person with chronic pain. The pain hindered the activities of daily living, decreased their desire to participate in exercise and social events. Unrelieved chronic pain may lead to decrease physical activities, depression, anxiety [21-22]. The pain was severe in around $60 \%$ of them and $90 \%$ was multiple regions, which most caused by back pain, arthritis, and neuroinflammation etl. Untreated chronic pain also caused sleep disturbances, malnutrition and various physical discomforts, most participants reported that they had poor sleep quality due to the pain. Pain and reduce mobility would lead to social isolation and depression and this is associated with cognitive dysfunction in many older people. The occurrence of chronic pain had also impacted on the participants' family and social lives. Obviously, these relationship interacted with each other, lack of sleep must influence the work and daily life, and then the motion and relationship, like that, it can induce a vicious cycle. It can significant reduced the quality of life, low level of happiness. If the team want to improve this, the essential and most important thing is to relief chronic pain. Based on the location, degree, pathogenesis, introduce the personalized guideline, at the same time, the team also introduce more effect scheme. Some research now even pointed that a robust predictive study to predict the transition from acute to chronic pain in a musculoskeletal trauma population [27]. Using such a comprehensive array of outcome measures will allow the development and validation of a predictive tool to predict development of chronic and disabling pain, and begin the process of identifying appropriate and precision interventions.

Encourage to have regular physical activity, view the chronic pain in a correct attitude. Physical activity could help to decrease peripheral vascular resistance, insulin resistance, and blood pressure, percentage of abdominal fat, cholesterol level and depression. The older adults have the greatest number of chronic diseases that may control or prevented their physical activity level. The older people investigated all live in the jumping-off area, lack of formal education and health-related knowledge. The majority of older people did not seek professional treatment, they thought the chronic pain is the normal signs with the age increased. Indeed, bodily pain has been identified as one of the common reasons for being physical inactive; thus leading a sedentary lifestyle among old persons, most older people went to mountains to find the herbal to pack on the painful region, some of the techniques were suggested by their friends or family. Uses of herbal remedies to reduce facial pain have been described, but there is generally insufficient evidence to support its use for chronic pain relief [3]. Because of little education, many people believed pain is the punishment by the god, their thinking were control by feudal thought, $61.9 \%$ people took the attitude to endure the pain, they endure as much as possible, worried to be burden of sons and daughters. So in this investigation, the older people are essential to change their thinking and attitude of chronic pain. Since a number of barriers exist to accessing care for chronic pain, the team should suggest the fundamental healthcare unit enforce to introduce the health education in the older people with chronic pain, as well as to help the older people to know more about chronic pain and improve the effectiveness of self-management.

Enforce self-management techniques Effective self-managers will feel confident in selecting the techniques that they believe will meet their specific needs at a given point of time and in a given environment of situation. Since the old people lack professional treatment sources due to the jumping-off area and poor education. They also had a number of other self-management to alleviate their chronic pain symptoms. Common strategies included application of Chinese herbal oil, self massage, cold or warm compression, using medical pads, nutrition, taking more rest, physical exercise and over-the-counter medications. As everyone knows, self-management is a very well behavior, however, the effective self-management is rooted in the correct knowledge. The management of chronic pain need the older people participated, promote and improve the ability of self-management, the final goal of pain management is the patients can control the pain by themselves effectively [23]. In 
the abstract of social support, except of physical and pharmacy strategies, the team found the older people did not want to mention their chronic pain to their family and friends because they did not want them to worry or they felt other would not understand their pain and could not help them. Especially their sons and daughters outside to earn money to support the family and they need to care their grandchildren, most of them choose to endure by themselves, never talked to the friends and family. So the research team recommend for the field of chronic pain, the predominant approach is holistic and multi-component, with many interventions, management of psychosocial consequences, social support, communication and life style changes.

\section{Conclusion}

$\mathrm{CP}$ are common in older people and are associated with postural instability, fear of falling, and increased incidence of falls, simultaneously, induced depression to some extent. Based on the study, mountain older people own their distinctive features to deal with the CP. However, CP has a multifactorial aetiology and complex dysfunction, therefore the old people should be encouraged to seek different ways to manage their pain, not just traditional but complementary and professional approaches. In such severely lacking professional high-level medical resource environment, the elder people should change their cognition of $\mathrm{CP}$ and choose pertinence approaches and instruments based on their own condition. For their own condition, the cost-effectiveness of the therapy is a crucial factor when choosing a certain product or technology. It has been considered the most suitable method to them is great important.

\section{References}

[1] Merskey H, Bogduk N, editor. Classification of chronic pain: descriptions of chronic pain syndromes and definitions of pain terms. Seattle: IASP Press; 1994. p. 210. Prepared by the Task Force on Taxonomy of the International Association for the Study of Pain.

[2] A big gap between town and country for supporting the elders [N]. YanJing night newspaper, 2013-07-12.

[3] Zhou Wei, Cui Ying, Yang Li. The health-realted quality life and affective factors of Midwest rural area during older people [J]. Chines Elder Journal, 2012, 32 (19):4252-4255.

[4] Sacks, Jeffrey J; Luo, Yao-Hua; Helmick, Charles G. Prevalence of specific types of arthritis and other rheumatic conditions in the ambulatory health care system in the United States, 2001-2005[J]. Arthritis Care Res (Hoboken):2010, 62(4):460-464.

[5] Levelle, S. G., Bean, J., Bandeen-Roche, K., et al. Musculoskeletal pain and risk for falls in older disabled women living in the community. Journal of the American Geriatrics Society 2002, 50:671-678.

[6] Jade Parker, The development of pain medicine in Italy and the rest of Europe 40 years after the first International
Association for the Study of Pain Congress. Pain Manag. 2017, 7 (1): 15-18.

[7] Richard Staelin, Sree N Koneru, Ian M Rawe. An over-the-counter central sensitization therapy: a chronic back pain registry study of pain relief, medication use and their adverse effects. Pain Manag. 2017, 7 (2): 99-111.

[8] L. J. Kong, H. S. Zhan, Y. W. Cheng, W. A. Yuan, B. Chen, M. Fang. Massage therapy for neck and shoulder pain: a systematic review and meta-analysis. Evid Based Complement Alternat Med: 2013: 613279.

[9] Johnson, Mark I; Hudson, Matt. Generalizing, deleting and distorting information about the experience and communication of chronic pain. Pain Manag. 2016, 6(5): 411-414.

[10] Tavel ME. Somatic symptom disorders without known physical causes: one disease with many names? Am. J. Med. 2015, 128(10), 1054-1058.

[11] Bjelland, Ingvar;Dahl, Alv A;Haug, Tone Tangen; Neckelmann, Dag. The validity of the Hospital Anxiety and Depression Scale. An updated literature review. J Psychosom Res. 2002, 52 (2): 69-77.

[12] Montorezi A, Vahdaninia M, Ebrahimi M, Jarvandi S. The Hospital Anxiety and Depression Scale (HADS): translation and validation study of the Iranian version. Health Qual Life Outcomes. 2003, 1:14.

[13] Herrero MJ, Blanch J, Peri JM, De Pablo J, Pintor L, Bulbena A. A validation study of Hospital Anxiety and Depression Scale (HADS) in a Spanish population. Gen. Hosp. Psychiatry 2003 (25): 277-283.

[14] Marcolino JAM, Mathias LAST, Piccinini Filho L, Guarantini AA, Suzuki FM, Alli LAC. Hospital Anxiety and Depression Scale: a study on the validation of the criteria and reliability on pre-operative patients. Rev. Bras. Anestesiol. 2007(57):5262 .

[15] Michopoulos I, Douzenis A, Kalkavoura C et al. Hospital Anxiety and Depression Scale (HADS): validation in a Greek general hospital sample. Ann. Gen. Psychiatry 2008(7): 4.

[16] Soh, G. Ang, H. G. Comparison of two pain rating scales among Chinese cancer patients [in English]. Chinese Medical Journal, 1992(105):953-956.

[17] Aun, C;Lam, Y M; Collett, B. Evaluation of the use of visual analogue scale in Chinese patients. Pain 1986(25): 215-221.

[18] Cleeland CS. The brief pain inventory user guide, https://www.mdanderson.org/education-and-research/departm ents-programs-and-labs/departments-and-divisions/symptom-r esearch/symptom-assessment-tools/BPI_UserGuide.pdf (accessed 17 March 2016).

[19] Nicholas, Michael K; Asghari, Ali; Blyth, Fiona M. What do the numbers means? Normative data in chronic pain measures. Pain 2008, 134(1-2): 158-173.

[20] Parmalee, P. A., Katz, I. R., Lawton, M. P. The relation of pain to depression among institutionalized aged. The Journals of Gerontology. Series B. Psychological Sciences, 1991, 46(1):15-21.

[21] Erdal, K J; Zautra, A J. Psychological impact of illness downturns: a comparison of new and chronic conditions. Psychology and Aging, 1995, 10(4): 570-577. 
[22] Turp JC, Gobetti JP. Trigeminal neuralgia-an update. Compend Contin Educ Dent 2000(21): 279-292.

[23] Barlow, Julie; Wright, Chris; Sheasby, Janice;Turner, Andy; Hainsworth, Jenny. Self-management approaches for people with chronic conditions: a review. Patient Educ Couns, 2002, 48(2): 177-187.

[24] Julie C. Kendall, Simon D. French, Jan Hartvigsen and Michael F. Azari. Chiropractic treatment including instrument-assisted manipulation for non-specific dizziness and neck pain in community-dwelling older people: a feasibility randomized sham-controlled trial. Chiropractic \& Manual Theropies, (2018) 26:14.

[25] Sobral APT, Godoy CLH, Fernandes KPS, et al. Photomodulation in the treatment of chronic pain in patients with temporomandibular disorder: protocol for cost-effectiveness analysis. BMJ Open 2018;8:eo18326.doi:10.1136/bmjopen-2017-018326.

[26] Damsgard E, Solqard H, Johannessen K, Wennevold K, Kvarstein G, Pettersen G, Garcia B. Understanding Pain and Pain Management in Elderly Nursing Home patients applying an interprofessional learning activity in health care students: a Norwegian Study. Pain Manag Nurs, 2018 May 17. pii:S 1524-9042(17)30395-8.doi:10.1016/j.pmn.2018.02.064.

[27] Rushton AB, Evans DW, Middlebrook N, et al. Development of a screening tool to predict the risk of chronic pain and disability following musculoskeletal trauma: protocol for a prospective observational study in the United Kingdom. BMJ Open 2018;8:e017876.doi:0.1136/bmjopen-2017-017876. 